\title{
Does interspecies hybridization affect the host specificity of parasites in cyprinid fish?
}

Andrea Šimková ${ }^{*}$, Martina Dávidová', Ivo Papoušek ${ }^{2,3}$ and Lukáš Vetešník

\begin{abstract}
Background: Host specificity varies among parasite species. Some parasites are strictly host-specific, others show a specificity for congeneric or non-congeneric phylogenetically related host species, whilst some others are nonspecific (generalists). Two cyprinids, Cyprinus carpio and Carassius gibelio, plus their respective hybrids were investigated for metazoan parasites. The aim of this study was to analyze whether interspecies hybridization affects host specificity. The different degrees of host specificity within a phylogenetic framework were taken into consideration (i.e. strict specialist, intermediate specialist, and intermediate generalist).

Methods: Fish were collected during harvesting the pond and identified using meristic traits and molecular markers. Metazoan parasite species were collected. Host specificity of parasites was determined using the following classification: strict specialist, intermediate specialist, intermediate generalist and generalist. Parasite species richness was compared between parental species and their hybrids. The effect of host species on abundance of parasites differing in host specificity was tested.
\end{abstract}

Results: Hybrids harbored more different parasite species but their total parasite abundance was lower in comparison with parental species. Interspecies hybridization affected the host specificity of ecto- and endoparasites. Parasite species exhibiting different degrees of host specificity for C. carpio and C. gibelio were also present in hybrids. The abundance of strict specialists of $C$. carpio was significantly higher in parental species than in hybrids. Intermediate generalists parasitizing C. carpio and C. gibelio as two phylogenetically closely related host species preferentially infected C. gibelio when compared to C. carpio, based on prevalence and maximum intensity of infection. Hybrids were less infected by intermediate generalists when compared to C. gibelio.

Conclusions: This finding does not support strict co-adaptation between host and parasite genotypes resulting in narrow host specificity, and showed that hybrid genotypes are susceptible to parasites exhibiting host specificity. The immune mechanisms specific to parental species might represent potential mechanisms explaining the low abundance of parasites in C. gibelio x C. carpio hybrids.

Keywords: Cyprinid fish, Interspecies hybridization, Metazoan parasites, Monogenea, Host specificity

\section{Background}

Host specificity is commonly defined by means of a simple classification into specialists or generalists. A specialist parasitizes a single host species, whilst a generalist parasitizes several host species [1]. However, because of the multifaceted nature of host specificity, this traditional classification applied in many studies can mask the differences in host specificity of the parasites that are associated to ecological importance, phylogenetic relatedness or

\footnotetext{
* Correspondence: simkova@sci.muni.cz

'Department of Botany and Zoology, Faculty of Science, Masaryk University, Kotláŕská 2, 611 37, Brno, Czech Republic

Full list of author information is available at the end of the article
}

geographical distribution of their host species [2]. Therefore, host specificity can be expressed at different scales (see $[2,3]$ ). Considering a phylogenetic framework, the semiquantitative classification of host specificity taking into account the phylogenetic relatedness of host species was applied for congeneric monogenean parasites by Desdevises et al. [4] and Šimková et al. [5]. A strict specialist (or species-specific parasite) is a parasite species infecting a single host species [1,5], whilst other specialists may infect phylogenetically closely related hosts such are congeneric hosts (termed as intermediate specialists by Desdevises et al. [4]) or some parasites (termed as 
intermediate generalists by Desdevises et al. [4]) may infect non-congeneric but phylogenetically closely related host species e.g. hosts forming a monophyletic group [5].

Up to now, only a few studies investigating whether or not host specificity is affected by interspecies hybridization, a common phenomenon in animals, have been performed [6,7]. These studies were limited to one parasite species, but in natural conditions the hosts are parasitized by various parasite species, and parasite communities on hosts are formed by both specialists and generalists. Concerning cyprinid fish, it is estimated that more than $30 \%$ are hybrids [8]. Currently, there is a lack of knowledge concerning host susceptibility to metazoan parasites in fish hybrids, and only two studies dealing with the host specificity of some metazoan parasites in hybridizing cyprinid fish have been performed $[9,10]$.

The susceptibility or resistance of hybrids to viral, protozoan or helminth infection was investigated mainly in mice within their hybrid zones. It was shown that mouse hybrids are more susceptible to infection than their parental species due to genomic incompatibilities in the introgressed genomes of the hybrids [11-13]. However, mice trapped in the same part of the hybrid zone had two different phenotypes, susceptible and resistant, but an unfavorable genetic combination occurred more frequently in recombinant genotypes, producing a higher susceptibility to parasitic helminths [12]. The study of co-infection by two species, one protozoan and one helminth with different life traits, led to the suggestion that hybrid susceptibility is applied only to parasites that exert adequate constraints on their host to induce the selection of co-adapted genes of resistance [14].

Several studies aimed at investigating hybrid resistance to viral infection were performed in fish under experimental conditions, suggesting (conversely to mice) that fish hybrids are less susceptible than their parental species (for instance $[15,16]$ ). The experimental study of salmonid hybrids showed that the abundance of two Gyrodactylus species (Monogenea) was lower on hybrids than on their respective pure-bred natural hosts, and a parental sire-and-dam influence on the resistance of hybrids was observed [17]. The highly frequent natural hybrids of two cyprinid species, Alburnus alburnus and Rutilus rubilio, from Lake Mikri Prespa (Northern Greece) showed a high susceptibility to metazoan parasites when compared with pure species [9]. However, the hybrids of two cyprinids, native Parachondrostoma toxostoma and invasive Chondrostoma nasus in southern France, were less parasitized by ecto- and endoparasites than both pure species in the localities where these fish species live in sympatry [10].

Generally, ectoparasites with a direct life cycle are considered to be more specific than endoparasites with a complex life cycle. Among fish ectoparasites, Monogenea, which live on the gills, fins and skin, represent a parasite group with narrow host specificity. The genetically based host-specific barriers occurring due to co-evolution between a host and its specific parasites can determine the presence of specific parasites. Host resistance to two congeneric monogenean parasites, each of them specific to one parental species, was found in laboratory-raised F1 hybrids of anurans [7]. However, parasite infection in hybrid specimens is also determined by the degree of hybridization, i.e. the introgression rate, as was shown for gill Diplozoon species (Monogenea), in hybridizing the cyprinid fish species Barbus barbus and B. meridionalis [6].

Among cyprinid species, the hybrids between Cyprinus carpio and Carassius gibelio have previously been identified using morphological criteria and molecular markers (for instance [18]). However, reports of hybrids living in areas where pure cyprinid species live in sympatry are rare, particularly if the hybrids are identified using only morphological criteria (unpublished study). Concerning the parasitofauna of C. carpio and C. gibelio, both harbor several species specific or genus specific monogenean species.

The aim of this study was to investigate the metazoan parasite communities in C. carpio and C. gibelio and their respective hybrids. We focused on metazoan parasites exhibiting the different degrees of host specificity to analyze whether interspecies hybridization affects host specificity. The aspect of phylogenetic relatedness of host species was included in host specificity delimitation.

\section{Methods}

For this study, 12 individuals of C. carpio, 14 individuals of C. gibelio and 13 individuals of their respective hybrids were investigated. All specimens were of the same age and were collected while harvesting the Hlohovecký fish pond (4846'51"N, 16²7'2"E; Danube River Basin, the Czech Republic) in November. All hybrid specimens found during the harvesting were collected. The hybrids were identified using meristic traits such as number of gill rakers, length of upper and lower barbels, and number of scales in the lateral line. All investigated fish were fin-clipped and the clips were stored in ethanol for molecular analyses. The analyses of five microsatellite markers and partial mtDNA representing the hypervariable part of the control region (D-loop) were performed for each individual fish following Papoušek et al. [19]. Microsatellite loci GF29, MFW2, MFW7, J1 and J62 were amplified with previously published primers (see $[19,20]$ ). Microsatellite and mitochondrial genotypes in C. carpio, C. gibelio and their respective hybrids are shown in Table 1 . The genetic differences in multilocus genotypes among two parental species and their respective hybrids were analyzed using principal coordinate analysis (PCoA) performed in GenAlEx 6.41 [21]. PCoA was performed using standardized genetic distances. 
Table 1 Microsatellite and mitochondrial genotypes in C. carpio, C. gibelio and their respective hybrids

\begin{tabular}{|c|c|c|c|c|c|c|}
\hline Fish & GF29 & MFW2 & MFW7 & J1 & $J 62$ & Haplotype \\
\hline Hybrid 1 & $197 / 257$ & $157 / 253$ & $179 / \mathbf{1 8 8}$ & $123 / 151$ & $158 / 178$ & C. gibelio \\
\hline Hybrid 2 & 197/251 & 157/239 & $175 / 258$ & $123 / 147$ & $156 / 178$ & C. gibelio \\
\hline Hybrid 3 & 197/273 & 157/213 & $175 / 258$ & $125 / 141$ & $148 / 176$ & C. gibelio \\
\hline Hybrid 4 & $203 / \mathbf{2 7 3}$ & $157 / 247$ & $179 / 258$ & $125 / 157$ & $158 / 178$ & C. gibelio \\
\hline Hybrid 5 & 197/255 & 157/237 & $179 / 258$ & $123 / 141$ & $148 / 178$ & C. gibelio \\
\hline Hybrid 6 & 193/255 & $157 / 265$ & $179 / 256$ & $125 / 159$ & $156 / 176$ & C. gibelio \\
\hline Hybrid 7 & 197/255 & $157 / 239$ & $179 / 258$ & $127 / 157$ & $162 / 178$ & C. gibelio \\
\hline Hybrid 8 & 197/269 & $157 / 253$ & $179 / 256$ & $123 / 157$ & $160 / 176$ & C. gibelio \\
\hline Hybrid 9 & 199/251 & $157 / 249$ & 169/196 & $123 / 159$ & $156 / 178$ & C. gibelio \\
\hline Hybrid 10 & 197/255 & 157/249 & 175/196 & $127 / 151$ & $160 / 178$ & C. gibelio \\
\hline Hybrid 11 & 199/255 & 157/249 & 153/196 & $123 / 151$ & $156 / 178$ & C. gibelio \\
\hline Hybrid 12 & 197/255 & $157 / 247$ & 179/192 & $123 / 151$ & $156 / 178$ & C. gibelio \\
\hline Hybrid 13 & 197/255 & $157 / 245$ & $173 / 242$ & $125 / 151$ & $156 / 180$ & C. gibelio \\
\hline C. carpio & 251-319 & $181-267$ & $192-258$ & $123-127$ & $154-160$ & C. carpio \\
\hline C. gibelio & 193-201 & 157 & 153-181 & 141-177 & $176-180$ & C. gibelio \\
\hline C. carpio * & 243-283 & 169-271 & $188-276$ & - & - & - \\
\hline C. gibelio * & 193-207 & 157 & $175-179$ & - & - & - \\
\hline C. gibelio ** & 195-201 & 157 & 175-195 & - & - & - \\
\hline
\end{tabular}

The allele size ranges according to Hänfling et al. [18] (shown with *) and Papoušek et al. [19] (shown with **) are included. Microsatellite alleles with presumed origin in C. carpio are shown in bold. The alleles outside the ranges of $C$. carpio or $C$. gibelio analyzed in this study are shown in italic.

Immediately after capture, the fish were transported live to the laboratory in barrels with the original oxygenated water and dissected within 24 hours. All fish were killed in the laboratory by severing the spinal cord. Standard length (in millimeters) and body weight (in grams) were recorded. Complete dissection of the fish was performed following the method of Ergens and Lom [22]. Fish were examined for all metazoan parasites following the standard techniques used in fish parasitology. All parasites were removed and fixed according to standard methods and then identified to species level using a light microscope (Olympus BX50) equipped with phasecontrast, differential interference contrast (DIC) and Digital Image Analysis (Olympus MicroImage ${ }^{\text {ma }}$ for Windows 95/98/NT 7.0 (Olympus Optical Co.)). ANOVA followed by multiple Tukey post hoc tests was used to test the differences in fish body length between groups (C. carpio, C. gibelio and hybrids). The host range (i.e. host specificity in Table 2) was primarily identified using Moravec [23] and Pugachev et al. [24]. For Paradilepis scolecina, the host range was completed using Scholz et al. [25]. Even if the internet sources NHM Parasite-Host Database (http://www. nhm.ac.uk/research-curation/scientific-resources/taxonomysystematics/host-parasites/database/index.jsp) and Gyrobase (http://www.gyrodb.net) suggest wider host range for several parasite species, we found the same host species as indicated by the above references after filtering the host species representing the accidental findings, questionable host records, host species found only in captivity, parasite misidentification or doubtful identification. In addition, we removed the host species restricted to Asia (especially to Southeast Asia and Baltic Sea) because our study was performed at the local level. We also highlight that the host specificity shown for each parasite species in Table 2 corresponds to the records of the most frequent host species included in NHM Parasite-Host Database (i.e. C. carpio, C. gibelio and C. carassius were the most frequently recognized host species for the parasite species exhibiting a certain degree of host specificity). For delimitation host specificity, we applied the semiquantitative classification developed for congeneric monogeneans by Desdevises et al. [4]: strict specialist living on a single host species, intermediate specialist living on congeneric host species (i.e. Carassius gibelio and Carassius carassius in our study), intermediate generalist living on non-congeneric host species forming a monophyletic group (i.e. Cyprinus carpio, Carassius gibelio and Carassius carassius in our study), generalist living on phylogenetically distant host species (i.e. parasitizing fish species of different subfamilies within Cyprinidae or different cyprinid and non-cyprinid fish species). This classification essentially combined the information from basic and phylogenetic host specificity (as defined by Poulin et al. [2]).

ANCOVA followed by multiple Tukey post hoc tests were applied to test the differences in parasite abundance between fish groups (C. carpio, C. gibelio and hybrids); standard fish 
Table 2 The presence of metazoan parasite species in C. gibelio, C. carpio and their respective hybrids

\begin{tabular}{|c|c|c|c|c|}
\hline Parasite species & Host specificity & CaGi & $\mathrm{CyCa}$ & Hybrids \\
\hline \multicolumn{5}{|l|}{ Monogenea } \\
\hline Dactylogyrus achmerovi & strict specialist (CyCa) & - & $100 \%(1-39)$ & $15.4 \%(1-2)$ \\
\hline Dactylogyrus anchoratus & intermediate generalist & $85.7 \%(1-19)$ & $16.7 \%(1-2)$ & $46.2 \%(1-10)$ \\
\hline Dactylogyrus dulkeiti & intermediate specialist & - & - & $7.7 \%(1)$ \\
\hline Dactylogyrus extensus & strict specialist (CyCa) & - & $83.3 \%(1-13)$ & $15.4 \%(1-2)$ \\
\hline Dactylogyrus formosus & intermediate specialist & $7.1 \%(2)$ & - & $7.7 \%(1)$ \\
\hline Dactylogyrus molnari & strict specialist (CyCa) & - & $100 \%(5-21)$ & $30.8 \%(1-4)$ \\
\hline Dactylogyrus intermedius & intermediate specialist & $28.6 \%(1-4)$ & - & - \\
\hline Dactylogyrus vastator & intermediate generalist & $14.3 \%(2-4)$ & - & $15.4 \%(1-2)$ \\
\hline Dactylogyrus spp. larvae & not evaluated & $50 \%(1-8)$ & $58.3 \%(1-9)$ & $46.2 \%(1-6)$ \\
\hline Gyrodactylus katharineri & generalist & $7.1 \%(1)$ & - & - \\
\hline Gyrodactylus longoacuminatus & intermediate generalist & $35.7 \%(3-84)$ & $8.33 \%(7)$ & $53.8 \%(1-10)$ \\
\hline Gyrodactylus medius & intermediate generalist & $7.1 \%(1)$ & $16.7 \%(2-6)$ & $7.7 \%(1)$ \\
\hline Gyrodactylus shulmani & intermediate generalist & $21.4 \%(9-118)$ & $16.7 \%(2-6)$ & $15.4 \%(1-4)$ \\
\hline Gyrodactylus sprostonae & intermediate generalist & $100 \%(2-4754)$ & $100 \%(3-500)$ & $92.3 \%(1-1017)$ \\
\hline Gyrodactylus vimbi & generalist & $7.1 \%(2)$ & - & - \\
\hline Eudiplozoon nipponicum & strict specialist (CyCa) & - & $58.3 \%(1-7)$ & $23.1 \%(1-3)$ \\
\hline \multicolumn{5}{|l|}{ Crustacea } \\
\hline Argulus foliaceus & generalist & $21.4 \%(1-2)$ & $75 \%(1-9)$ & $46.2 \%(1-2)$ \\
\hline \multicolumn{5}{|l|}{ Hirudinea } \\
\hline Piscicola geometra & generalist & - & - & $7.7 \%(1)$ \\
\hline \multicolumn{5}{|l|}{ Digenea } \\
\hline Diplostomum spathaceum larvae & generalist & $21.4 \%(1)$ & $58.3 \%(2-48)$ & $69.2 \%(1-9)$ \\
\hline Echinostomatidae fam.sp. larvae & not evaluated & - & - & $7.7 \%(1-2)$ \\
\hline Paryphostomum radiatum larvae & generalist & $7.1 \%(1)$ & - & - \\
\hline \multicolumn{5}{|l|}{ Cestoda } \\
\hline Khawia sinensis & strict specialist (CyCa) & - & $25 \%(1-5)$ & $15.4 \%(1-2)$ \\
\hline Paradilepis scolecina & generalist & $50 \%(1-7)$ & - & $7.7 \%(1-10)$ \\
\hline \multicolumn{5}{|l|}{ Nematoda } \\
\hline Nematoda sp. & not evaluated & $7.1 \%(1)$ & - & $7.7 \%(1-2)$ \\
\hline
\end{tabular}

The prevalence and intensity of infection (min-max) are included. CaGi - Carassius gibelio, CyCa - Cyprinus carpio.

body length was used as covariate. Parasite abundance was log-transformed prior to ANCOVA. KW ANOVA followed by multiple comparison tests were used to compare the differences in abundance of two generalist parasite species between fish groups because in this case the distribution of parasite abundance did not fit a normal distribution. All analyses were performed using Statistica 10.0 for Windows, StatSoft Inc. The similarity between parasite communities was calculated using the qualitative Jaccard index on the parasite presence/absence data. The Jaccard index ranges from zero (no species is common between two host groups) to one (two host groups share the same parasite species).

This study was approved by animal care and use committee in Faculty of Science, Masaryk University in Brno (Czech Republic).

\section{Results}

All investigated specimens within each host group had a similar body size (standard length $240.07 \pm 13.24 \mathrm{~mm}$ for C. gibelio, $323.08 \pm 31.23 \mathrm{~mm}$ for C. carpio and $288 \pm 32.47 \mathrm{~mm}$ for their respective hybrids). ANOVA revealed a significant effect of host on standard body length $\left(\mathrm{F}_{2,36}=30.75, \mathrm{p}<0.001\right)$. The standard body length of C. carpio was greater than that of C. gibelio $(\mathrm{p}<0.001)$. The standard body length of hybrids was intermediate between C. gibelio $(\mathrm{p}<0.001)$ and C. carpio $(\mathrm{p}=0.013)$. The analyses of mtDNA showed that all hybrid specimens analyzed in this study possessed the haplotype of C. gibelio (Table 1), which indicated that the hybrids were descended from C. gibelio maternally. Microsatellite analysis of 13 putative hybrid specimens revealed the 
presence of nine alleles in the GF29 locus, nine alleles in the MFW2 locus, 11 alleles in the MFW7 locus, eight alleles in the J1 locus, and eight alleles in the J62 locus. For each of the loci GF29, MFW2, MFW7 and J1, one allele corresponded to C. carpio and the other to C. gibelio (based either on alleles actually observed or on size ranges described in Hänfling et al. [18] or Papoušek et al. [19] for all putative hybrids) (Table 1). With respect to locus J62, the alleles in two hybrid specimens (Hybrids 3 and 5) with a presumed origin from $C$. carpio were slightly outside the observed size range. The PCoA plot visualized the genetic differentiation between $C$. carpio, $C$. gibelio and the hybrids (Figure 1). The first two axes explained $63.57 \%$ of total variability.

A total of 23 metazoan parasite species were found in a whole fish sample including 15 species of Monogenea, 1 species of Crustacea, 1 species of Hirudinea, 3 species of Digenea, 2 species of Cestoda and 1 species of Nematoda (Table 2). Hybrids harbor more different parasite species than each of the parental species i.e. a total of 19 parasite species were found in hybrids whilst C. carpio harbor 12 and C. gibelio 15 parasite species. The similarity in parasite component communities between hybrids and parental species, based on the presence-absence of parasite species, was higher between hybrids and C. carpio (0.632) than between hybrids and C. gibelio (0.478). The similarity in parasite communities between $C$. carpio and $C$. gibelio was low (0.35).

The host specificity of each parasite species found on fish in our study is included in Table 2. Following the published data, 14 from 23 parasite species showed a certain degree of host specificity (using the semiquantitative classification of Desdevises et al. [4] for delimitation of host specificity). We identified strict specialists parasitizing solely $C$. carpio; intermediate specialists parasitizing $C$. gibelio and $C$. carassius; intermediate generalists parasitizing Carassius species (C. gibelio and/or C. carassius) and C. carpio. 13 from 14 parasites exhibiting a certain degree of host specificity measured in the phylogenetic context belong to Monogenea and one to Cestoda. Four monogenean parasites (3 Dactylogyrus species and Eudiplozoon nipponicum) and the cestode parasite Khawia sinensis were strictly host specific for $C$. carpio. In our fish sample, Dactylogyrus and Gyrodactylus parasites represented the genera with the highest species richness. All Dactylogyrus species and four Gyrodactylus species exhibited a certain degree of host specificity on the basis of above criteria (Table 2). Each parasite exhibiting a certain degree of host specificity (except for D. intermedius) was recorded in hybrids.

A significant effect of host on total parasite abundance was found (ANCOVA, whole model $\mathrm{F}_{3,35}=3.60, \mathrm{p}=0.023$ with standard length $\mathrm{F}=2.61, \mathrm{p}=0.115$ and host $\mathrm{F}=5.28$, $\mathrm{p}=0.010$ ). The total parasite abundance in hybrids tended to be lower when compared to both parental species (Figure 2). Multiple Tukey post hoc tests revealed a significant difference in parasite abundance between hybrids and C. gibelio ( $\mathrm{p}=0.019$ ). However, no significant difference in total parasite abundance between hybrids and $C$. carpio $(\mathrm{p}=0.409)$, or between C. gibelio and C. carpio $(\mathrm{p}=0.311)$ was found.

The prevalence and intensity of infection of each Cyprinus carpio specific parasite species (i.e. Dactylogyrus achmerowi, D. extensus, D. molnari, Eudiplozoon nipponicum and Khawia sinensis) were higher in C. carpio than in hybrids (Table 2). ANCOVA revealed a significant effect of host on the abundance of strict specialists (whole model $\mathrm{F}_{2,22}=$ 59.57, $\mathrm{p}<0.001$, body length $\mathrm{F}=3.32, \mathrm{p}=0.082$, host $\mathrm{F}=$ $73.14, \mathrm{p}<0.001)$ with $C$. carpio being significantly more parasitized than hybrids (Figure 3A). ANCOVA using only strictly specific monogenean parasites (i.e. after removing Khawia sinensis) revealed the same result (whole model $\mathrm{F}_{2,22}=54.70, \mathrm{p}<0.001$, body length $\mathrm{F}=2.23, \mathrm{p}=0.150$, host $\mathrm{F}=69.77, \mathrm{p}<0.001)$. The prevalence and intensity of infection of intermediate specialists (i.e. the parasite species potentially infecting congeneric hosts - C. gibelio and $C$. carassius) were low in $C$. gibelio and hybrids

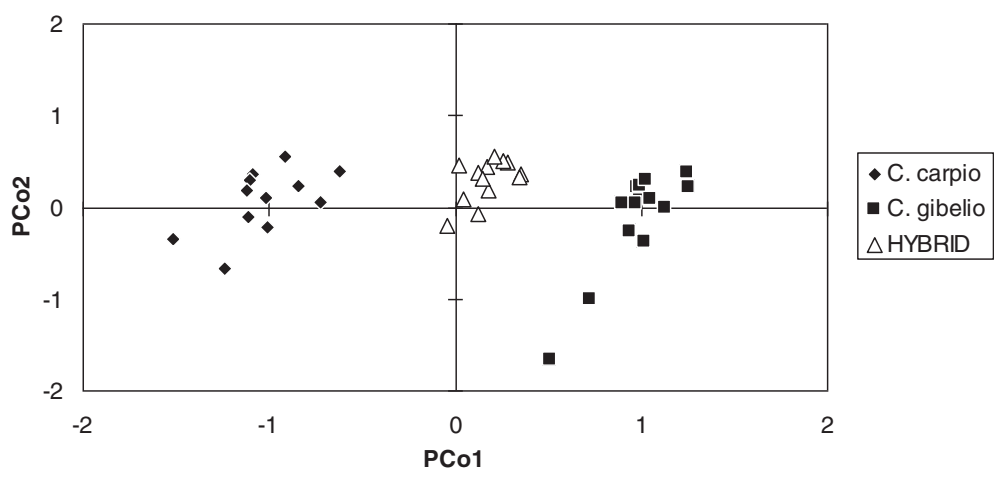

Figure 1 The genetic differentiation of multilocus genotypes among C. carpio, C. gibelio and their respective hybrids based on principal coordinate analysis. 


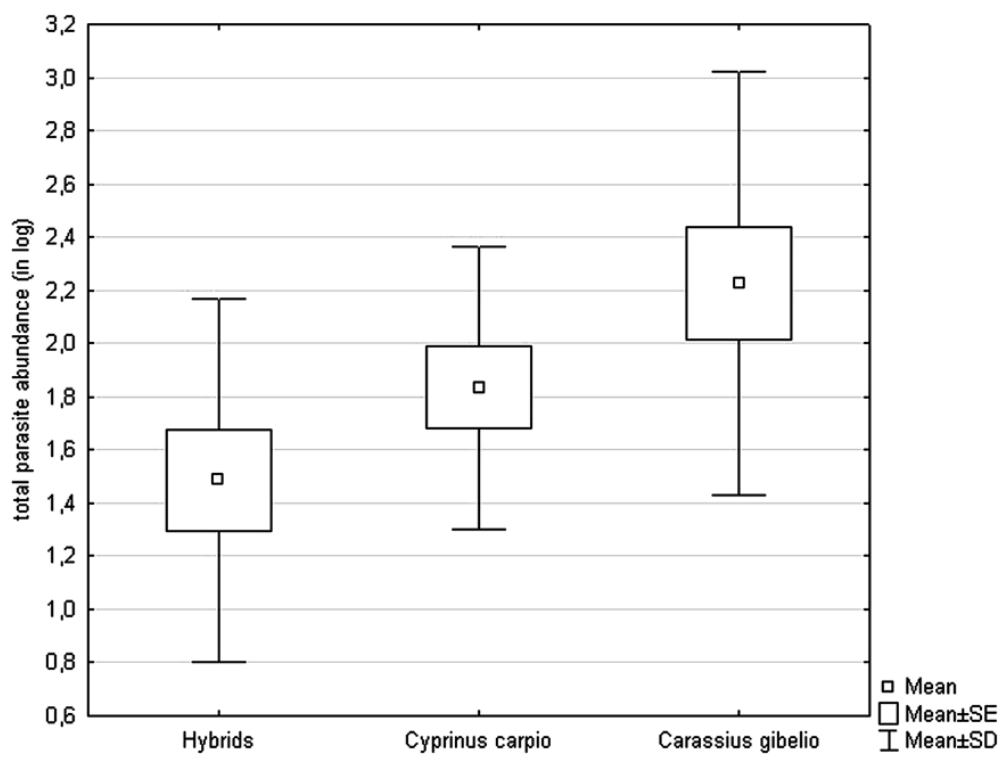

Figure 2 Parasitism in C. carpio, C. gibelio and their hybrids measured by total parasite abundance.

(Table 2) and, moreover, D. intermedius was present solely in C. gibelio. Concerning intermediate generalists (i.e. D. anchoratus, D. vastator, G. longoacuminatus, G. medius, G. shulmani and G. sprostonae), they preferentially infected C. gibelio compared with $C$. carpio based on prevalence and intensity infection values (Table 2). The only exception from this pattern was G. medius, which is however considered as host specific for C. carpio by Moravec [23] and Pugachev et al. [24]. Considering intermediate generalists exhibiting the specificity for phylogenetically closely related hosts, ANCOVA revealed the significant effect of host on the parasite abundance (whole model $F_{3,35}=3.59, p=0.023$, body length $F=1.93$, $\mathrm{p}=0.174$, host $\mathrm{F}=4.71, \mathrm{p}=0.015)$. Tukey post hoc test revealed the significantly higher abundance of intermediate generalists in C. gibelio when compared to hybrids $(\mathrm{p}=0.023)$ and there was also the trend for higher abundance in $C$. gibelio when compared to $C$. carpio (even if the difference was not statistically significant as revealed by $\mathrm{p}=0.079$ ) (see Figure $3 \mathrm{~B}$ ). The same results were found when removing G. medius from the analyses.

Concerning generalist parasites, the majority of them achieved low prevalence and intensity of infection values (Table 2). Such generalists were rarely found in C. gibelio and/or in hybrids. On the other hand, two generalist parasites Argulus foliaceus and Diplostomum spathaceum were present in C. carpio, C. gibelio and their hybrids. The prevalence of $A$. foliaceus was higher in $C$. carpio when compared to prevalence of this parasite species in $C$. gibelio and hybrids, whilst the prevalence of $D$. spathaceum was higher in hybrids when compared to the prevalence of this species in C. carpio and C. gibelio (Table 2). However, a significant difference in the abundance of these two parasite species was found only between parental species (for $A$. foliaceus KW ANOVA $\mathrm{H}_{2,39}=9.02, \mathrm{p}=0.011$, multiple comparison $\mathrm{p}=0.019$ and for $D$. spathaceum KW ANOVA $\mathrm{H}_{2,39}=8.61, \mathrm{p}=0.014$, multiple comparison $\mathrm{p}=0.033$ ) with $C$. carpio being more parasitized than C. gibelio.

\section{Discussion}

The two fish species investigated in the present study, C. gibelio and C. carpio, are phylogenetically related cyprinids, probably of the same origin and historical dispersion [26]. In nature, they live in sympatry, which can promote their hybridization. The hybrids of $C$. gibelio and C. carpio analyzed in our study were confirmed using both meristic traits and molecular identification. All of the hybrid specimens bore the haplotype of $C$. gibelio, according to the analysis of mitochondrial DNA, and all of them expressed one allele of $C$. gibelio and one allele of C. carpio, according to microsatellite analysis. Thus, this suggests that all of them are F1 offspring of female C. gibelio and male C. carpio. Moreover, all of them express the same morphology.

Two principal outcomes result from our study. The first one indicates that hybrids are able to harbor more different species of parasites than each of their parental species. The majority of parasite species belong to Monogenea with Dactylogyrus and Gyrodactylus representing a large part of parasite diversity. However, the total abundance of parasites tended to be lower in hybrids when compared to C. carpio and C. gibelio. Thus, the interspecies hybrids probably have a lower susceptibility to at least some metazoan parasites when compared to their parental species, leading to low total parasite abundance in hybrids. A 

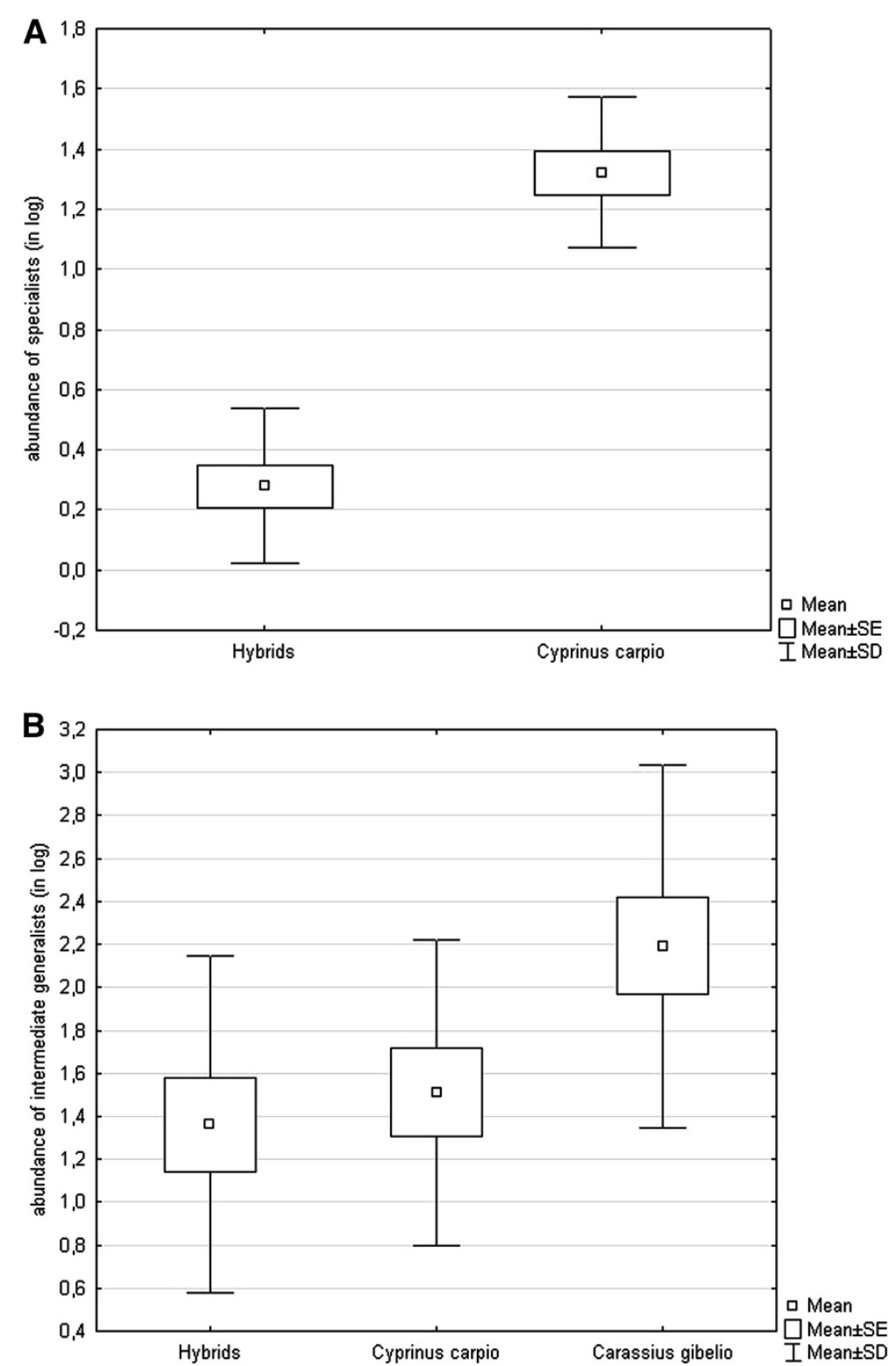

Figure 3 The effect of interspecies hybridization on host specificity for (A) strict specialists in C. carpio and hybrids of $C$. carpio and C. gibelio, (B) intermediate generalists in C. carpio, C. gibelio and their hybrids.

similar observation of low parasite abundance in interspecies fish hybrids was reported in an experimental study of infection by two Gyrodactylus species, G. salaris and G. derjavini, in pure-bred Salmo salar and Salmo trutta and their half-sib hybrids [17]. The study suggests that hybrids in nature may act as a reservoir for gyrodactylids, potentially supporting a wider diversity of species than their parental species and disseminating gyrodactylids of both host species. Our findings support this hypothesis as we showed such a pattern of infection for many monogenean species and several endoparasite species.

Contrary to our findings, a study of hybridization between two cyprinid species, Alburnus alburnus and Rutilus rubilio from Lake Mikri Prespa, Northern Greece, showed that the hybrids were highly susceptible to metazoan parasite infection including Dactylogyrus and Diplozoon (Monogenea), Bolbophorus confusus (larval stages of Digenea) and Pomphorhynchus bosniacus (Acanthocephala) [9]. This was explained by the different spatial and trophic position of hybrids, making them more exposed to parasites. Alternatively, it was proposed that hybrids could contain attractive substances from each of their parental species in their mucus or that perhaps immune defense expressed in parental mucus is impaired in hybrids [9]. The cyprinid species C. gibelio and C. carpio investigated in our study have the same spatial and trophic position, but their mucus substances or other 
immune mechanisms specific to the parental species might possibly represent potential mechanisms explaining the low abundance of parasites in C. gibelio x C. carpio hybrids. The studies performed with hybrid mice also show findings similar to those concerning fish hybrids described by Dupont and Crivelli [9], i.e. natural hybrids of mice are more infested by helminth parasites than both parental taxa [11,12], suggesting that the co-adapted gene systems controlling parasite infection are broken down in hybrid genomes because of high gene introgression.

The next outcome of our study was that hybridization between two phylogenetically closely-related non-congeneric cyprinid species affects the host specificity of metazoan parasites. Each of the two cyprinid species analyzed harbor specific parasite fauna, and especially specific monogenean species. C. carpio harbor strictly specific parasites, whilst C. gibelio harbor intermediate specialists, i.e. parasites that were previously recognized as specific to the congeneric hosts $C$. gibelio and C. carassius. In addition, these cyprinid species harbor the group of parasites exhibiting wider host specificity measured in phylogenetic context. The host range of such intermediate generalists was limited to phylogenetically closely-related C. carpio, C. gibelio and C. carassius. Concerning the host specificity of the most diverse parasite genera in C. carpio and C. gibelio, oviparous gill monogeneans of Dactylogyrus showed a high level of host specificity, i.e. each Dactylogyrus species was either strictly host specific or showed specificity for congeneric or non-congeneric phylogenetically related cyprinid species (as was shown for a large sample of Dactylogyrus species parasitizing cyprinid fish by Šimková et al. [5]). On the other hand, viviparous gill and skin monogenean parasites of Gyrodactylus showed low level of host specificity, i.e. the majority of Gyrodactylus species were intermediate generalists, and two of them were generalists infecting phylogenetically distant fish species. We showed that hybrids harbor the parasites exhibiting a certain level of host specificity.

Our study also shows that parasite life strategy, i.e. ectoparasitism versus endoparasitism, is not a feature limiting the effect of interspecies hybridization on host specificity, because Khawia sinensis, a cestode species, which is on the basis of published helminthological collections from the field studies (see review of Moravec [23]) considered as host specific to C. carpio, was also found in hybrids. However, the occurrence of this common carp-specific endoparasite in hybrid specimens cannot be explained by different feeding habitats between C. gibelio and C. carpio, as suggested by Dupont and Crivelli [9] (see above). It is more likely that the hybrids acquired some immune mechanisms specific to each pure host species, which facilitated the presence of both specific ecto- and endoparasites; however, because of co-adaptation, which is expected between the pure host genotype and specific parasite genotype, infection by specific parasites in interspecies hybrids is low.
Our study showed that the abundance of all strictly specific common carp parasites was lower in hybrids than in pure species. In the case of intermediate generalists exhibiting phylogenetic specificity, both parental species C. gibelio and $C$. carpio, as well as their hybrids, were susceptible to infection and a significant effect of host on the parasite abundance of intermediate generalists was found when taking host body size into account. We showed that the prevalence and maximum intensity of infection of such parasites were higher in C. gibelio than in C. carpio, which indicates that $C$. gibelio could be considered as their preferred host (Šimková et al. [5] suggested that even a generalist parasite may prefer some host species within its host range) The infection by intermediate generalists in hybrids was lower when compared to the preferred C. gibelio or some intermediate generalists even tended to reach the values intermediate between parental species. Thus, host susceptibility traits for specific parasites are probably genetically controlled and a low susceptibility can be transferred as a dominant trait through interspecific crosses between different cyprinid species. However, such a hypothesis needs to be tested in the future. We cannot support strict co-adaptation between the host genotype of a pure species and a specific parasite genotype potentially explaining narrow host specificity. On the other hand, the hybrids investigated in our study seem to bear "favorable" recombinant genotypes associated with a low level of infection by specific parasites but facilitating their transmission. A similar finding, i.e. low infection by metazoan parasites (especially Monogenea), was found for hybrids of P. toxostoma and C. nasus from the Durance and Ardeche (South France) sympatric zones [10]. However, in their study, both the $P$. toxostoma genotype and the recombinant genotypes of hybrids were less susceptible to Dactylogyrus parasites when compared to the pure $C$. nasus genotype, suggesting co-evolutionary interactions between $C$. nasus and their Dactylogyrus species.

Dupont and Crivelli [9] showed that interspecies cyprinid hybrids from Lake Mikri Prespa harbor all Dactylogyrus host specific to the parental species and also that Dactylogyrus parasitizing species other than parental species were present in hybrids at low intensity. However, in their study, the pattern of high parasite infection by dominant host-specific Dactylogyrus was similar in the hybrids and pure species. This discrepancy between different hybridizing cyprinid systems could imply a different level of gene introgression (high gene introgression might induce the break-down of a coadaptation gene system leading to high parasite infection in hybrids [12]), or it might be related to the different frequencies of hybrids in two systems (i.e. rare favorable C. gibelio $x$ C. carpio hybrid genotypes versus frequent unfavorable R. rubilio and A. alburnus hybrid genotypes). Therefore, further ecological and genetic studies could be helpful in interpreting the pattern of parasite infection in hybridizing fish species. 
Our study showed that even the infection by several generalist parasites found in all fish groups - C. carpio, C. gibelio and their hybrids - was shifted toward one parental species (i.e. the crustacean parasite Argulus foliaceus and the digenean parasite Diplostomum spathaceum preferentially infected C. carpio). This may suggest the function of some host species-specific immune mechanisms regulating also the infection of generalist parasites between different cyprinid species.

\section{Conclusions}

The interspecies hybridization of two non-congeneric phylogenetically closely-related cyprinid species C. carpio and C. gibelio affects the host specificity of their metazoan parasite species, i.e. the parasites exhibiting a certain degree of host specificity occur in hybrid specimens. Strict specialists showed a preference for parental species when compared with hybrids, likely resulting from the co-adaptation of host and parasite genotypes. However, on the basis of prevalence and maximum intensity of infection, the majority of intermediate generalists exhibited a preference for $C$. gibelio (probably also determined genetically) when compared to C. carpio, likely resulting in intermediate infection values for some parasite species in recombinant genotypes of F1 hybrids compared to parental host genotypes or low infection values for other parasite species found in both hybrids and C. carpio.

\section{Competing interests}

The authors declare that they have no competing interests.

\section{Authors' contributions}

AŠ designed the study and drafted the manuscript. AŠ, MD and LV participated in the field studies to acquire fish and parasite data. MD determined the parasites. IP carried out the molecular analyses and statistical analysis of molecular data. AŠ analyzed the whole data. AŠ, MD, LV and IP were involved in drafting the manuscript or revising it critically for important intellectual content. All authors read and approved the final version of the manuscript.

\section{Acknowledgements}

The field work performed in 2010 was funded by the Czech Science Foundation, Project No. 524/07/0188. Other analyses in this study and the three authors, $A \breve{S}, M D$ and $L V$, were funded or supported by the Czech Science Foundation, Project No. P505/12/0375. We are very grateful to Matthew Nicholls for English revisions to the final version.

\section{Author details}

${ }^{1}$ Department of Botany and Zoology, Faculty of Science, Masaryk University, Kotlářská 2, 611 37, Brno, Czech Republic. ${ }^{2}$ Institute of Vertebrate Biology, Academy of Sciences of the Czech Republic, v.v.i., Květná 8, 603 65, Brno, Czech Republic. ${ }^{3}$ Department of Biology and Wildlife Diseases, Faculty of Veterinary Hygiene and Ecology, University of Veterinary and Pharmaceutical Sciences Brno, Palackého tř. 1/3, 612 42, Brno, Czech Republic.

Received: 19 February 2013 Accepted: 6 April 2013

Published: 12 April 2013

\section{References}

1. Euzet $L$, Combes $C$ : Les problèmes de l'espèce chez les animaux parasites. Mem Soc Zool Fr 1980, 40:239-285.
2. Poulin R, Krasnov BR, Mouillot D: Host specificity in phylogenetic and geographic space. Trends Parasitol 2011, 27:355-361.

3. Pojmanska T, Niewiadomska K: New trends in research on parasite host specificity: a survey of current parasitological literature. Ann Parasitol 2012, 58:57-61.

4. Desdevises Y, Morand S, Legendre P: Evolution and determinants of host specificity in the genus Lamellodiscus (Monogenea). Biol J Linn Soc 2002, 77:431-443.

5. Šimková A, Verneau O, Gelnar M, Morand S: Specificity and specialisation of congeneric monogeneans parasitizing cyprinid fish. Evolution 2006, 60:1023-1037.

6. Le Brun N, Renaud F, Berrebi P, Lambert A: Hybrid zones and host-parasite relationships: effect on the evolution of parasitic specificity. Evolution 1992, 46:56-61.

7. Jackson JA, Tinsley RC: Parasite infectivity to hybridising host species: a link between hybrid resistance and allopolyploid speciation? Int $J$ Parasitol 2003, 33:137-144.

8. Scribner KT, Page KS, Bartron ML: Hybridization in freshwater fishes: a review of case studies and cytonuclear methods of biological inference. Rev Fish Biol Fisher 2000, 10:293-323.

9. Dupont F, Crivelli AJ: Do parasites confer a disadvantage to hybrids? A case study of Alburnus alburnus $x$ Rutilus rubilio, a natural hybrid of Lake Mikri Prespa, Northern Greece. Oecologia 1988, 75:587-592.

10. Šimková A, Navrátilová P, Dávidová $M$, Ondračková $M$, Sinama M, Chappaz R, Gilles A, Costedoat C: Does invasive Chondrostoma nasus shift the parasite community structure of endemic Parachondrostoma toxostoma in sympatric zones? Parasites and Vectors 2012, 5:200.

11. Sage RD, Heynemen D, Lim KC, Wilson AC: Wormy mice in hybrid zone. Nature 1986, 324:60-63.

12. Moulia C, Aussel JP, Bonhomme F, Boursot P, Nielsen JT, Renaud F: Wormy mice in a hybrid zone: a genetic control of susceptibility to parasite infection. J Evol Biol 1991, 4:679-687.

13. Derothe JM, Le Brun N, Loubes C, Perriat-Sanguinet M, Moulia C: Susceptibility of natural hybrids between house mouse subspecies to Sarcocystis muris. Int J Parasitol 2001, 31:15-19.

14. Derothe JM, Loubes C, Perriat-Sanguinet M, Orth A, Moulia C: Experimental trypanosomiasis of natural hybrids between house mouse subspecies. Int J Parasitol 1999, 29:1011-1016.

15. LaPatra SE, Lauda KA, Jones GR, Shewmaker WD, Groff JM, Routledge D: Susceptibility and humoral response of brown trout $\mathrm{x}$ lake trout hybrids to infectious hematopoietic necrosis virus: a model for examining disease resistance mechanisms. Aquaculture 1996, 146:179-188.

16. Hedrick RP, Waltzek TB, McDowell TS: Susceptibility of koi carp, common carp, goldfish, and goldfish $\mathrm{x}$ common carp hybrids to cyprinid herpesvirus-2 and herpesvirus-3. J Aquat Anim Health 2006, 18:26-34.

17. Bakke TA, Soleng A, Hartus PD: The susceptibility of Atlantic salmon (Salmo salar L.) x brown trout (Salmo trutta L.) hybrids to Gyrodactylus salaris Malmberg and Gyrodactylus derjavini Mikailov. Parasitology 1999, 119:467-481.

18. Hänfling B, Bolton P, Harley M, Carvalho GR: A molecular approach to detect hybridisation between crucian carp (Carassius carassius) and nonindigenous carp species (Carassisus spp. and Cyprinus carpio). Freshwater Biol 2005, 50:403-417.

19. Papoušek I, Vetešník L, Halačka K, Lusková V, Humpl M, Mendel J: Identification of natural hybrids of gibel carp Carassius auratus gibelio (Bloch) and crucian carp Carassius carassius (L.) from lower Dyje River floodplain (Czech Republic). J Fish Biol 2008, 72:1230-1235.

20. Yue $\mathrm{GH}$, Orban L: Polymorphic microsatellites from silver crucian carp (Carassius auratus gibelio Bloch) and cross-amplification in common carp (Cyprinus carpio L). Mol Ecol Notes 2002، 2:534-536.

21. Peakall R, Smouse PE: GENALEX 6: genetic analysis in excel. Population genetic software for teaching and research. Mol Ecol Notes 2006, 6:288-295.

22. Ergens R, Lom J: Causative agents of fish diseases. Prague: Academia; 1970 (in Czech).

23. Moravec F: Checklist of Metazoan Parasites of Fishes of the Czech Republic and the Slovak Republic (1873-2000). Prague: Academia; 2001.

24. Pugachev ON, Gerasev PI, Gussev AV, Ergens R, Khotenowsky I: Guide to Monogenoidea of freshwater fish of Palaeartic and Amur regions. Milano: Ledizioni-Ledipublishing; 2010. 
25. Scholz T, Bray RA, Kuchta R, Řepová R: Larvae of gryporhynchid cestodes (Cyclophyllidea) from fish: a review. Folia Parasitol 2004, 51:131-152.

26. Durand JD, Tsigenopoulos CS, Ünlü E, Berrebi P: Phylogeny and biogeography of the family Cyprinidae in the Middle East inferred from cytochrome $b$ DNA - evolutionary significance of this region. Mol Phyl Evol 2002, 22:91-100.

doi:10.1186/1756-3305-6-95

Cite this article as: Šimková et al.: Does interspecies hybridization affect the host specificity of parasites in cyprinid fish? Parasites \& Vectors 2013 6:95.

\section{Submit your next manuscript to BioMed Central and take full advantage of:}

- Convenient online submission

- Thorough peer review

- No space constraints or color figure charges

- Immediate publication on acceptance

- Inclusion in PubMed, CAS, Scopus and Google Scholar

- Research which is freely available for redistribution 